\title{
Baroreflex sensitivity and neurohormonal activation in patients with acute myocardial infarction
}

\author{
Juha Hartikainen, Frej Fyhrquist, Kari Tahvanainen, Esko Länsimies, Kalevi Pyörälä
}

\begin{abstract}
Objective-To examine the relationship between baroreflex sensitivity and neurohormonal activation in patients with an acute myocardial infarction.

Methods-Baroreflex sensitivity, plasma noradrenaline, atrial natriuretic factor, endothelin-1, and plasma renin activity were measured in 37 male patients about 10 days after their first myocardial infarction, and in 15 healthy controls. Baroreflex sensitivity was assessed from the regression line relating the change in $R R$ interval to the change in systolic blood pressure following an intravenous bolus injection of phenylephrine. The measurements were repeated after a follow up of three months.
\end{abstract}

Results-There was a significant inverse correlation between baroreflex sensitivity and plasma noradrenaline measured before hospital discharge $(r=-0.43, P<$ 0.01). Patients with increased plasma noradrenaline ( $\geqslant 2 S D$ above the mean of the age matched control group) had significantly lower baroreflex sensitivity than patients with normal plasma noradrenaline (8.7 (SD 4.6) v $12.1 \quad(6 \cdot 1)$ $\mathrm{ms} / \mathrm{mm} \mathrm{Hg}, P<0.05)$. The change in baroreflex sensitivity during the follow up showed a significant inverse correlation with the change of plasma noradrenaline $(r=-0.450, \quad P<0.01)$. Furthermore, when patients with increased plasma noradrenaline before hospital discharge were analysed separately, baroreflex sensitivity at three months in patients in whom plasma noradrenaline had decreased to normal values was significantly higher than in patients in whom plasma noradrenaline had remained increased $(14.6(5.7)$ v $8.1(8 \cdot 1) \mathrm{ms} / \mathrm{mm}$ Hg, $P<0.05)$. On the other hand, baroreflex sensitivity was not related to the levels of plasma atrial natriuretic factor, plasma endothelin-1, or plasma renin activity. Neither was any relationship found between change in baroreflex sensitivity and change in plasma atrial natriuretic factor, endothelin-1, or plasma renin activity during the follow up.

Conclusions-The impairment baroreflex sensitivity after myocardial infarction was associated with increased concentration of plasma noradrenaline, that is, sympathetic activation, but not with plasma atrial natriuretic factor, endothelin-1, or plasma renin activity. Baroreflex sensitivity provides information about cardiac vagal control as well as about the balance of cardiac sympatheticparasympathetic regulation.

(Br Heart f 1995;74:21-26)

Keywords: atrial natriuretic factor; autonomic nervous system; catecholamines; endothelin-1; myocardial infarction; renin

Acute myocardial infarction results in an impairment of cardiac autonomic regulation ${ }^{12}$ as well as in a wide spectrum of neurohormonal activation, including increased plasma noradrenaline, ${ }^{34}$ atrial natriuretic factor, ${ }^{5}$ endothelin-1, ${ }^{6}$ and renin activity. ${ }^{7}$ Impairment of cardiac autonomic regulation, that is, low baroreflex sensitivity, in patients with recent myocardial infarction, has been found to be related to poor prognosis because of cardiac electrical instability leading to ventricular tachyarrhythmias and sudden cardiac death. ${ }^{8-10}$ Neurohormonal activation in patients with myocardial infarction has also been associated with complications and excessive mortality. ${ }^{11-14}$

The mechanism responsible for the impairment of baroreflex sensitivity in patients with acute myocardial infarction has not yet been defined. It has not been found to be related to size, type, or location of myocardial infarction, nor to the severity of cardiac dysfunction. ${ }^{8915}$ One possibility is that impairment of baroreflex sensitivity might be associated with neurohormonal activation. So far, however, there are no data available on this subject. Thus the objective of this study was to evaluate the relationship between baroreflex sensitivity and neurohormonal activation in patients with acute myocardial infarction.

\section{Methods}

SUBJECTS AND STUDY PROTOCOL

The study population consisted of 37 men aged 53.8 (SD 9.2) years (range 32-68) recovering from their first myocardial infarction. Myocardial infarction was diagnosed according to the criteria of the World Health Organisation. ${ }^{16}$ The location of myocardial infarction was anterior in 16 patients (46\%) and inferior in 12 patients $(30 \%)$. Twenty two patients $(61 \%)$ had $Q$ wave infarction and 15 
patients $(29 \%)$ were treated with thrombolytic therapy. $\beta$ Blockers were used by 34 patients $(94 \%)$, calcium channel blockers by five $(14 \%)$, and oral nitrates by $31(84 \%)$. Medication was not changed during the follow up. Patients with diabetes mellitus, Parkinson's disease, chronic alcoholism, and atrial fibrillation were excluded. In addition, a control group of 15 age matched healthy male volunteers not belonging to hospital personnel was recruited. The mean age of the controls was 54.6 (9.1) years (range 37-67).

Baroreflex sensitivity was determined before discharge from the hospital, about 10 days after the myocardial infarction. Before the measurement of baroreflex sensitivity, blood samples were taken for the analysis of plasma noradrenaline, atrial natriuretic factor, endothelin-1, and plasma renin activity. In addition, left ventricular ejection fraction was assessed by echocardiography. The measurement of baroreflex sensitivity and hormone analyses were repeated three months after the myocardial infarction. The control group was studied with the same protocol as patients, with the exception that hormone analyses were performed only once.

The ethics committee of Kuopio University Hospital approved the study protocol and informed consent was obtained from all patients and controls.

ASSESSMENT OF BAROREFLEX SENSITIVITY

Baroreflex sensitivity was evaluated with a modification of the method described by Smyth et al. ${ }^{17}$ In brief, the method was as follows. Phenylephrine $150 \mu \mathrm{g}$ was injected into an antecubital vein to produce a rapid increase of blood pressure and a concomitant reduction in heart rate. The bolus injection was repeated three times with 10 minute intervals. ECG and continuous non-invasive arterial blood pressure signals (Finapres, Ohmeda) were recorded and were then converted to digital signals with a temporal resolution of $200 \mathrm{~Hz} /$ channel and an amplitude resolution of 12 bits. The converted signals were stored in a microcomputer and analysed with a menu driven software package (Cafts, Medikro Oy). ${ }^{18}$

For the assessment of baroreflex sensitivity, beat to beat values of $R-R$ intervals (RRI) were plotted against the systolic arterial pressure values (SAP) of the preceding cardiac cycle (RRI(i) $v$ SAP(i-1)) during a period of increase in blood pressure. A linear regression analysis between RRI(i) and $\operatorname{SAP}(\mathrm{i}-1)$ was performed and the slope of the regression line and the corresponding Pearson correlation coefficient were calculated. Slopes with correlation coefficients of $r \geqslant 0.80$ or statistically significant $(P<0.05)$ were accepted. The mean value of the accepted slopes represented baroreflex sensitivity.

To assess the reproducibility of the assessment of baroreflex sensitivity, measurements carried out in the control group with three month intervals were compared. The two measurements of baroreflex sensitivity showed a high correlation $(r=-0.903, \mathrm{P}<0.001)$ and the coefficient of variation (the standard deviation of the mean difference divided by the absolute mean of the two measurements $\times 100)^{19}$ was $7 \cdot 4 \%$.

\section{PLASMA HORMONE ANALYSES}

Blood samples for the plasma hormone analyses were taken after an overnight fast through a Teflon cannula in an antebrachial vein. After the insertion of the cannula the patients rested in the supine position for 30 minutes. Blood was drawn into prechilled tubes and placed on ice. Plasma was separated within 20 minutes at $4^{\circ}$, frozen immediately, and stored at $-70^{\circ} \mathrm{C}$ until assay. Plasma noradrenaline concentrations were analysed by high performance liquid chromatography (HPLC) com- के bined with electrochemical detection. ${ }^{20} 21$ Radioimmunoassay techniques described elsewhere were used for the assessment of $\vec{\omega}$ concentrations of plasma atrial natriuretic factor, ${ }^{22}$ endothelin-1, ${ }^{23}$ and plasma renin activity. ${ }^{24}$

LEFT VENTRICULAR EJECTION FRACTION

Left ventricular ejection fraction was evaluated with an Aloka SSD-870 ultrasonic sys- $\vec{c}$ tem. $M$ mode echocardiography recordings were performed from the left ventricular $\vec{\bullet}$ parasternal long axis view according to the of recommendation of the American Society of Echocardiography. ${ }^{25}$ Measurements obtained from five cardiac cycles were averaged.

\section{STATISTICAL METHODS}

Comparisons between different groups were performed with two tailed Student's $t$ test and analysis of covariance (using age as covariate). Paired $t$ tests were used for testing the significances of changes of baroreflex sensitivity and hormone levels during the follow up. Correlation analyses were performed using a least squares linear regression analysis and multivariate regression analysis. A $P$ value of $<0.05$ was considered statistically significant. The data are expressed as mean (SD) unless otherwise indicated.

\section{Results}

BAROREFLEX SENSITIVITY AT HOSPITAL DISCHARGE AND THREE MONTHS AFTER MYOCARDIAL INFARCTION

In patients with myocardial infarction, baroreflex sensitivity at hospital discharge and at three months was significantly lower than in the controls $(P<0.05$; table 1$)$. When the whole patient group was considered, no significant change in baroreflex sensitivity was found during the three month follow up. However, the change in baroreflex sensitivity during the follow up showed marked interindividual variation.

PLASMA HORMONE LEVELS AT HOSPITAL DISCHARGE AND THREE MONTHS AFTER MYOCARDIAL INFARCTION

Table 1 shows that plasma noradrenaline concentration was higher in patients than in controls at hospital discharge $(P<0.001$; fig 1$)$ as 
Table 1 Baroreflex sensitivity and neurohormonal activation in controls $(n=15)$ and in patients with a myocardial infarction $(M I)(n=37)$. Values are means $(S D)$.

\begin{tabular}{|c|c|c|c|}
\hline & \multirow[b]{2}{*}{ Controls } & \multicolumn{2}{|l|}{ MI patients } \\
\hline & & Discharge & 3 months \\
\hline $\begin{array}{l}\text { BRS }(\mathrm{ms} / \mathrm{mm} \mathrm{Hg}) \\
\text { Plasma noradrenaline }(\mathrm{mmol} / \mathrm{l}) \\
\text { Plasma ANF }(\mathrm{pg} / \mathrm{ml}) \\
\text { Plasma endothelin }(\mathrm{pg} / \mathrm{ml}) \\
\text { Plasma renin activity }(\mathrm{ng} / \mathrm{ml})\end{array}$ & $\begin{array}{c}16 \cdot 4(9 \cdot 7) \\
1 \cdot 5(0 \cdot 4) \\
126 \cdot 1(45 \cdot 8) \\
2 \cdot 2(1 \cdot 4) \\
2 \cdot 3(1 \cdot 0)\end{array}$ & $\begin{aligned} & 10 \cdot 2(5 \cdot 5)^{\star} \\
& 2.9(1 \cdot 6)^{\star \star} \\
& 175 \cdot 7(86 \cdot 4)^{\star} \\
& 4 \cdot 2(1 \cdot 8)^{\star \star} \\
& 1.9(1 \cdot 5)\end{aligned}$ & $\begin{array}{c}12 \cdot 0(7 \cdot 6)^{\star} \\
2 \cdot 6(1 \cdot 4)^{\star \star} \\
152 \cdot 7(84 \cdot 8) \dagger \\
3 \cdot 0(1 \cdot 0)^{\star}+\dagger \\
1 \cdot 9(1 \cdot 3)\end{array}$ \\
\hline
\end{tabular}

ANF, atrial natriuretic factor; BRS, baroreflex sensitivity. Measurements before hospital discharge (Discharge) and 3 months after MI ( 3 months). $\star \mathrm{P}<0.05 ; \star \star \mathrm{P}<0.001 v$ controls (two tailed Student's t test); $+\mathrm{P}<0.05 ; \mathrm{H}+\mathrm{P}<0.001 v$ hospital discharge (paired $t$ test). infarction. No significant change was observed in plasma noradrenaline during the follow up. Patients had higher concentrations of plasma atrial natriuretic factor than the control group at hospital discharge $(\mathrm{P}<$ $0 \cdot 05)$, but not at three months. Plasma atrial natriuretic factor decreased significantly during the follow up. Plasma endothelin-1 before hospital discharge was also higher in patients than in controls $(P<0.001)$. It decreased during the three month period, but still remained higher than in the controls at three months after myocardial infarction $(P<$ $0.05)$. Plasma renin activity in controls and patients did not differ at hospital discharge or at three months. No significant change in plasma renin activity was found during the follow up.

\section{RELATIONSHIP BETWEEN BAROREFLEX}

SENSITIVITY AND PLASMA HORMONES

Baroreflex sensitivity measured before hospital discharge showed an inverse correlation with plasma noradrenaline in univariate analysis $(r=-0.43, \mathrm{P}<0.01$, fig 2$)$. This correlation remained significant $(P<0.05)$ when adjusted for age, size, location and type of infarction, thrombolytic therapy, medication, resting heart rate, resting blood pressure, and left ventricular ejection fraction. In order to study the relationship between baroreflex sensitivity and plasma noradrenaline more comprehensively patients were divided into two

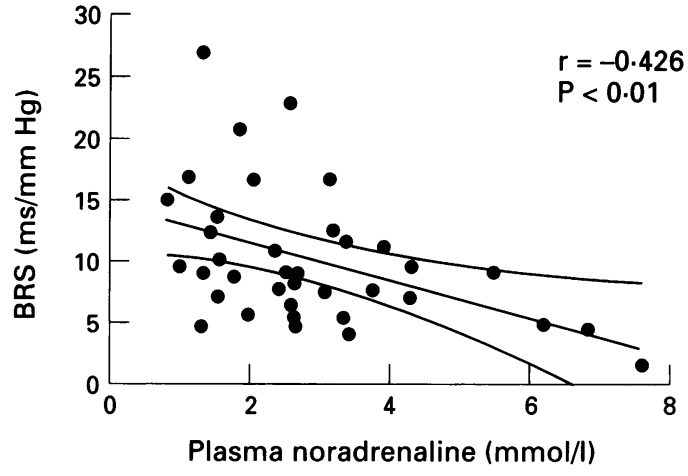

Figure 2 The relationship between baroreflex sensitivity (BRS) and plasma noradrenaline concentration in patients with acute myocardial infarction before the hospital discharge. The regression line and $95 \%$ confidence intervals are shown.

groups by using $2 \mathrm{SD}$ above the mean plasma noradrenaline for the controls $(2.3 \mathrm{nmol} / \mathrm{l})$ as the cut off value (fig 1 ). Accordingly, patients with plasma noradrenaline $\geqslant 2.3 \mathrm{nmol} / 1$ were considered as a group with increased plasma noradrenaline, and those with values of $<2 \cdot 3$ $\mathrm{nmol} / \mathrm{l}$ as a group with normal plasma noradrenaline. Patients with increased plasma noradrenaline had lower baroreflex sensitivity than patients with normal plasma noradrenaline $(P<0.05$, table 2$)$.

The change in baroreflex sensitivity showed an inverse correlation with the change in plasma noradrenaline during the three month follow up in univariate analysis $(r=-0 \cdot 45, \mathrm{P}<$ 0.01 , fig 3 ), as well as in multivariate analysis adjusting for age $(P<0.01)$. In addition, we studied in more detail the relationship between changes in baroreflex sensitivity and plasma noradrenaline in patients with increased plasma noradrenaline before hospital discharge. Patients in whom plasma noradrenaline decreased to normal values during the follow up had higher baroreflex sensitivity at three months than patients in whom plasma noradrenaline remained persistently elevated $(14.6(5 \cdot 7)$ v $8 \cdot 1(8 \cdot 1) \mathrm{ms} / \mathrm{mm} \mathrm{Hg}$, $P<0.05$, fig 4), that is, normalisation of
Figure 1 Plasma noradrenaline in healthy controls and in patients with acute myocardial infarction before their hospital discharge. Filled circles represent values for individuals and empty circles represent means of the controls and patients. Error bars = SEM. The dotted line shows $2 S D$ above the mean plasma noradrenaline of the controls $(2 \cdot 3$ nmolll) Plasma noradrenaline was considered increased if it exceeded $2 \cdot 3 \mathrm{nmoll}$ and normal if it was below $2 \cdot 3$ nmolll. $+P<0.001$.

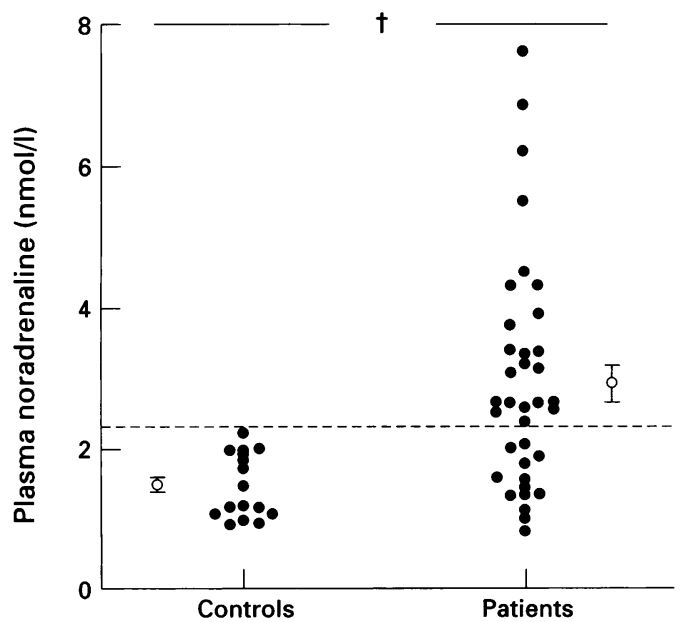

Table 2 The relationship between baroreflex sensitivity and neurohormonal activation before hospital discharge in patients with acute myocardial infarction. Values are means $(S D)$.

\begin{tabular}{lc}
\hline & $B R S(\mathrm{~ms} / \mathrm{mm} \mathrm{Hg})$ \\
\hline Plasma noradrenaline & \\
Normal & $12 \cdot 1(6 \cdot 1)^{\star}$ \\
Increased & $8 \cdot 7(4 \cdot 6)$ \\
Plasma ANF & $10 \cdot 5(6 \cdot 1)$ \\
$\quad$ Normal & $9 \cdot 2(3 \cdot 8)$ \\
Increased & $10 \cdot 3(5 \cdot 5)$ \\
Plasma endothelin-1 & $9 \cdot 5(5 \cdot 7)$ \\
$\quad$ Normal & $10 \cdot 5(5 \cdot 6)$ \\
Increased & $6 \cdot 9(2 \cdot 6)$ \\
Plasma renin activity & Normal \\
Increased & \\
\hline
\end{tabular}

ANF, atrial natriuretic factor; BRS, baroreflex sensitivity. Plasma noradrenaline, ANF, endothelin, and plasma renin activity were divided into two groups (normal and increased) at $2 \mathrm{SD}$ above the mean hormone activity of the control group. ${ }^{\star} \mathrm{P}<0.05 v$ the group with increased plasma noradrenaline (analysis of covariance). 
Figure 3 The relationship between the change in baroreflex sensitivity (BRS) and change in plasma noradrenaline during three months of follow up after myocardial infarction. The regression line and $95 \%$ confidence intervals are shown.

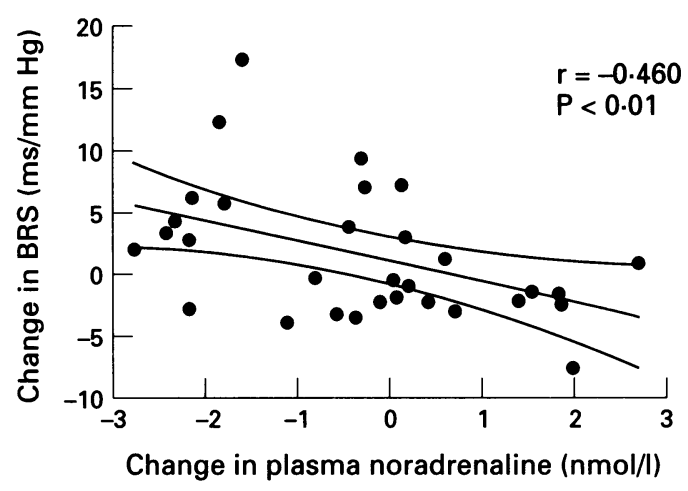

Figure 4 Baroreflex sensitivity (BRS) and plasma noradrenaline measured three months after myocardial infarction in patients with increased plasma noradrenaline $(\geqslant 2.3$ nmolll) before hospital discharge. The patients were divided into two groups; patients in whom plasma

noradrenaline had returned to normal during the three months (O) and patients with persistently increased plasma noradrenaline ( $\mathrm{O}$ ) at 3 months. Error bars = SEM. ${ }^{\star} P<0.05$.

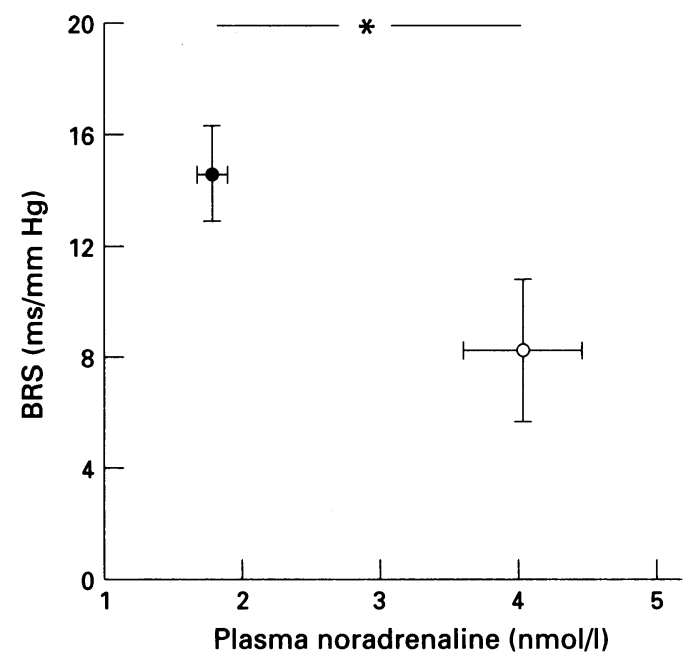

plasma noradrenaline was associated with a recovery of baroreflex sensitivity.

Plasma atrial natriuretic factor, endothelin1, and plasma renin activity did not show any significant correlations with baroreflex sensitivity, either in univariate or in multivariate analyses. When patients were dichotomised at 2 SD above the mean of the control group $(218 \mathrm{ng} / \mathrm{ml}$ for plasma atrial natriuretic factor, $5.0 \mathrm{pg} / \mathrm{ml}$ for plasma endothelin-1, and 4.3 $\mathrm{ng} / \mathrm{ml}$ for plasma renin activity) baroreflex sensitivity in patients with increased and normal levels of hormone activity did not differ from each other (table 2). Neither were any significant correlations observed between the change in baroreflex sensitivity and the change in plasma atrial natriuretic factor, endothelin-1, or plasma renin activity during the follow up.

\section{Discussion}

In this study we have shown that the impairment of baroreflex sensitivity after myocardial infarction is associated with sympathetic activation. Patients with increased plasma noradrenaline as a marker of sympathetic activation showed lower baroreflex sensitivity than patients with normal plasma noradrenaline. Furthermore, we found that in the whole patient group, as well as in patients with increased sympathetic activity at hospital discharge, a decrease in plasma noradrenaline during the follow up was accompanied by a recovery of baroreflex sensitivity.

According to current opinion, reflex changes in heart rate in response to baroreceptor stimulation are primarily due to changes in cardiac vagal activation, with only minor contribution from the sympathetic nervous system, ${ }^{26}$ that is, baroreflex sensitivity has been considered to be predominantly an indicator of cardiac parasympathetic regulation. ${ }^{17}$ Noradrenaline released from sympathetic nerve endings has been found to be the main source of plasma noradrenaline. Accordingly, plasma noradrenaline has been considered to reflect the level of efferent sympathetic activation. ${ }^{27} 28$ Thus our finding that baroreflex sensitivity was related to plasma noradrenaline was somewhat surprising.

One possible explanation for this finding is that, although changes in vagal activity predominate in the bradycardic reflex response, the effect of vagal stimulation on heart rate depends on the background sympathetic activity $^{29}$ - the higher the sympathetic tone the more depressed the baroreflex sensitivity. According to this theory, the depression of baroreflex sensitivity associated with myocardial infarction would be ascribed to increased level of sympathetic activity.

Secondly, it has been shown that, as well as controlling cardiac vagal activity, the baroreflex regulates efferent sympathetic activity: stimulation of arterial baroreceptors causes withdrawal of sympathetic outflow to skeletal muscles. ${ }^{30}$ Thus it can be assumed that impairment of baroreflex sensitivity following myocardial infarction results in attenuation of the tonic inhibitory signals responsible for the reduction of efferent sympathetic activity. As a consequence, the level of tonic sympathetic activity becomes increased. In this case impairment of the baroreflex would be the mechanism responsible for the increased plasma noradrenaline after myocardial infarction.

A third possibility is that baroreflex sensitivity and plasma noradrenaline are not directly dependent on each other, but that they share a common underlying determinant which accounts for the concomitant impairment of the baroreflex and increased sympathetic activity in patients with acute myocardial infarction. Indeed, it has been speculated that cardiac remodelling after myocardial infarction might promote excitation of cardiac sympathetic nerve endings and that this sympathetic afferent excitation could be responsible for the impairment of baroreflex sensitivity as well as for the increased efferent sympathetic activity. ${ }^{31}$

Whatever the mechanism, it seems to be obvious that baroreflex sensitivity provides information about cardiac vagal control as well as about sympathetic-parasympathetic balance. The association between baroreflex sensitivity and plasma noradrenaline is also logical from the clinical point of view that in patients with acute myocardial infarction both low baroreflex sensitivity ${ }^{89}$ and high concentrations of plasma noradrenaline ${ }^{4}$ have been 
shown to be related to excessive mortality caused by cardiac arrhythmias.

We did not find any significant relationship between baroreflex sensitivity and plasma concentrations of atrial natriuretic factor, endothelin-1, or plasma renin activity, neither was the change in baroreflex sensitivity during the three month follow up related to changes in plasma atrial natriuretic factor, endothelin1, or plasma renin activity. Previous studies on patients with acute myocardial infarction have shown that the level of plasma atrial natriuretic factor, endothelin-1, and renin activity correlate with the extent of left ventricular dysfunction. ${ }^{35-7}$ On the other hand, earlier studies have failed to show any significant relationship between baroreflex sensitivity and cardiac systolic function in patients with myocardial infarction. ${ }^{89} 15$ The concept that the mechanism of impairment of baroreflex sensitivity and plasma atrial natriuretic factor activation in myocardial infarction is different is further reinforced by the fact that, although both low baroreflex sensitivity and raised plasma atrial natriuretic factor are related to increased mortality, the mechanism of death seems to be different; patients with increased plasma atrial natriuretic factor tend to die because of heart failure, ${ }^{11}$ whereas mortality associated with low baroreflex sensitivity is not ascribed to heart failure but to ventricular arrhythmias and sudden cardiac death. ${ }^{8-10}$

\section{LIMITATIONS OF THE STUDY}

The study consisted of patients with uncomplicated myocardial infarction, that is, the patients had no clinical signs of heart failure or unstable angina pectoris. This constitutes a selection bias by excluding severely ill patients, in whom the most marked neurohormonal activation would be expected. Nevertheless, significant neurohormonal activation, especially in plasma noradrenaline, was observed. In addition, we suppose that this bias underestimates rather than overestimates the relationship between baroreflex sensitivity and plasma noradrenaline.

Medical treatment of the patients was based on clinical criteria. Medication was not altered during the follow up. The beneficial effect of $\beta$ blocker therapy in the postinfarction population is well established. ${ }^{32}$ Thus in our study a great majority of patients were on $\beta$ blocker treatment. In addition, most of the patients received antianginal medication, either oral nitrates ( 31 patients) or calcium channel blockers (five patients). In previous studies $\beta$ blockers have not had a consistent effect on baroreflex sensitivity or its recovery. ${ }^{33-36}$ We are not aware of studies reporting the effect of nitrate therapy on baroreflex sensitivity, but calcium channel blockers have been reported to have no significant effect. ${ }^{37}$ In our study plasma noradrenaline remained a significant determinant of baroreflex sensitivity when adjusted for medication. Thus it is very unlikely that medication had an important effect on the relationship between baroreflex sensitivity and plasma noradrenaline.
IMPLICATIONS OF THE STUDY

The impairment of cardiac autonomic regulation associated with acute myocardial infarction, as well as cardiac autonomic regulation in general, is a complex phenomenon. Cardiac function is influenced by both sympathetic and parasympathetic nervous systems and particularly by the interaction between the two. The complexity becomes evident in this study, in which baroreflex sensitivity, an index traditionally used to quantify cardiac parasympathetic regulation, was found to be related to plasma noradrenaline, a marker of sympathetic activation. This does not belie the concept that heart rate changes caused by baroreceptor stimulation are primarily the result of changes in cardiac parasympathetic activity. However, we do suggest that baroreflex sensitivity is not entirely divorced from sympathetic nervous system activity. Accordingly, instead of interpreting baroreflex sensitivity as a pure indicator of cardiac parasympathetic regulation, it should be considered as a marker of cardiac sympatheticparasympathetic interaction.

\section{CONCLUSIONS}

In conclusion, the impairment of baroreflex sensitivity as a consequence of myocardial infarction was found to be associated with increased sympathetic activation, but not with the concentrations of plasma atrial natriuretic factor, endothelin-1, or plasma renin activity. We hypothesise that, in addition to cardiac vagal regulation, baroreflex sensitivity provides information about cardiac sympatheticparasympathetic interaction.

This study was supported by Paavo Nurmi Foundation for cardiovascular research and a research fellowship of The European Society of Cardiology.

1 Ryan C, Hollenberg M, Harvey DB, Gwynn R. Impaired parasympathetic responses in patients after myocardial infarction. Am $\mathcal{F}$ Cardiol 1976;37:1013-8.

2 Webb SW, Adgey AAJ, Pantridge JF. Autonomic disturbance at onset of acute myocardial infarction. $B M \mathcal{\jmath}$ 1972;iii:89-92.

3 Sigurdson A, Held P, Swedberg K. Short- and long-term neurohormonal activation following acute myocardial infarction. Am Heart $\mathcal{F}$ 1993;126:1068-76.

4 McDonald L, Baker C, Bray C, McDonald A, Restieaux N. Plasma catecholamines after cardiac infarction. Lancet 1969;ii:1021-3.

5 Fontana F, Bernardi P, Spagnolo N, Capelli M. Plasma atrial natriuretic factor in patients with acute myocardial infarction. Eur Heart $\mathcal{F}$ 1990;11:779-87.

6 Tomoda H. Plasma endothelin-1 in acute myocardial infarction with heart failure. Am Heart $\mathcal{f}$ 1993;125: 667-72.

7 Vaughan DE, Lamas GA, Pfeffer MA. Role of left ventricular dysfunction in selective neurohumoral activation in the recovery phase of anterior wall acute myocardial infarction. Am ₹ Cardiol 1990;66:529-32.

8 La Rovere MT, Specchia G, Mortara A, Schwartz PJ. Baroreflex sensitivity, clinical correlates and cardiovascular mortality among patients with a first myocardial cular mortality among patients with a first myocardial
infarction. A prospective study. Circulation 1988;78: infarction.

9 Farrell TG, Odemuyiwa O, Bashir Y, Malik M, Ward DE, Camm AJ. Prognostic value of baroreflex sensitivity testing after acute myocardial infarction. Br Heart $\mathcal{F} 1992$ 67:129-37.

10 Hohnloser SH, Klingenheben T, van de Loo A, Hablawetz E, Schwartz PJ. Reflex versus tonic vagal activity as a prognostic parameter in patients with sustained ventricular tachycardia or ventricular fibrillation. Circulation 1994;89:1068-73.

11 Svanegaard J, Angelo-Nielsen K, Pindborg T. Plasma concentration of atrial natriuretic peptide at admission and risk of cardiac death in patients with acute myocardial infarction. Br Heart $₹$ 1992;68:38-42. 
12 Omland T, Aarsland T, Aakvaag A, Terje R, Dickstein K. Prognostic value of plasma atrial natriuretic factor, norepinephrine and epinephrine in acute myocardial infarction. Am $\mathcal{F}$ Cardiol 1993;72:255-9.

13 Lechleitner P, Genser N, Maier J, Artner-Dworzak E Dienstl F, Puschendorf B. Endothelin-1 in patients with complicated and uncomplicated myocardial infarction. Clin Invest 1992;70:1070-2.

14 Vaney C, Waeber B, Turini GA, Marpalith D, Brunne HR, Perret C. Renin and the complications of acute myocardial infarction. Chest 1984;86:40-3.

15 Hartikainen J, Mäntysaari M, Mussalo H, Tahvanainen K, Länsimies E, Pyörälä K. Baroreflex sensitivity in men with recent myocardial infarction; impact of age. Eur Heart 7 1994;15:1512-9.

16 World Health Organization. MONICA Manual. Geneva: WHO, 1990.

17 Smyth HS, Sleight P, Pickering GW. Reflex regulation of arterial pressure during sleep in man: a quantitative method of assessing baroreflex sensitivity. Circ Res 1969 24:109-21.

18 Tahvanainen $\mathrm{K}$, Länsimies E, Tikkanen $\mathrm{P}$, Hartikainen J, Kärki T, Lyyra T, et al. Microcomputer-based monitoring of cardiovascular functions in simulated microgravity Adv Space Res 1992;12:227-36.

19 Bland JM. An introduction to medical statistics. Oxford: Oxford University Press, 1989:277-96.

20 Nyyssönen K, Parviainen MT. Plasma catecholamines: laboratory aspects. Crit Rev Clin Lab Sci 1989;27: 211-36.

21 Nyyssönen K, Parviainen MT. Practical observations and sources of error in assays of plasma catecholamines by high-performance liquid chromatography with electromechanical detection. Clin Chem 1987;33 1938-9.

22 Tikkanen I, Fyhrqvist F, Metsärinne K, Leidenius R Plasma atrial natriuretic peptide in cardiac disease and during infusion in healthy volunteers. Lancet 1985;ii: 66-9.

23 Fyhrquist F, Metsarinne K, Saijonmaa O, Tikkanen I, Rosenlöf $K$, Tikkanen $T$. Raised plasma endothelin-1 concentration following cold pressor test. Biochem Concentration following cold press

24 Fyhrquist F, Soveri P, Puutula L, Stenman U-H.
Radioimmunoassay of plasma renin activity. Clin Chem 1976;22:250-6.

25 Sahn DJ, DeMaria A, Kisslo J, Weyman A Recommendations regarding quantification of $\mathrm{M}$-mode echocardiographic measurements. Circulation 1978;58: 1072-83.

26 Robinson BF, Epstein SE, Beiser D, Braunwald E. Control of heart rate by the autonomic nervous system. Circ Res 1966;19:400-11.

27 Goldstein DS, McCarty R, Polinsky RJ, Kopin IJ. Relationship between plasma norepinephrine and sympathetic neural activity. Hypertension 1983;5:552-9.

28 Wallin BG, Sundlof G, Eriksson BM, Dominiak $P, \frac{T}{D}$ Grobecker $\mathrm{H}$, Lindblad LE. Plasma noradrenaline correlates to sympathetic muscle nerve activity in $c$ normotensive man. Acta Physiol Scand 1981;111:69-73.

29 Levy MN. Sympathetic-parasympathetic interactions in $\overrightarrow{\overline{\vec{F}}}$ the heart. Circ Res 1971;29:437-45.

30 Ferguson DW, Berg WJ, Roach PJ, Oren RM, Mark AL. Effects of heart failure on baroreflex control of sympathetic neural activity. Am f Cardiol 1992;69:523-31.

31 Schwartz PJ, La Rovere MT, Vanoli E. Autonomic nervous system and sudden cardiac death. Circulation $\mathbb{\nabla}$ 1992;85(suppl I):I-77-91.

32 Yusuf S, Peto R, Lewis J, Collins R, Sleight P. Beta block- ڤ ade during and after myocardial infarction: An overview of the randomized trials. Prog Cardiovasc Dis 1985;17: $\overrightarrow{0}$ 335-71.

33 Ogawa J, Takata $S$, Aoki K, Oukuwa $H$, Iwase N, Ikeda $T$, $\overrightarrow{.}$ et al. Acute changes in the properties of baroreflexes in $\omega$ man after beta-blockade. Clin Exp Pharmacol Physiol man after beta-

34 Takeshita A, Matsuguchi H, Nakamura M. Effect of coronary occlusion on arterial baroreflex control of heart $I$ rate. Cardiovasc Res 1980;14:303-6.

35 Odemuyiwa O, Farrell T, Staunton A, Sneddon J, i Poloniecki J, Bennett $\mathrm{D}$, et al. Influence of thrombolytic therapy on the evolution of baroreflex sensitivity after 음 myocardial infarction. Am Heart f 1993;125:285-91.

$36 \mathrm{La}$ Rovere MT, Mortara A, Capomolla S, Cobelli F, $\vec{C}$ Schwartz PJ. Autonomic reflexes and beta-blockade in $ᄃ$ post-MI patients (abstr). Eur Heart $\mathcal{F}$ 1990;11:1394.

37 Parati G, Mancia G. Calcium antagonists in the treatment of arterial hypertension. Am Heart $₹$ 1993;125:642-8. 